\title{
The efficacy and safety of immune checkpoint inhibitors combined with antiangiogenic drugs in renal cell carcinomas: a systematic review and meta-analysis
}

\author{
Qian He, Jiayi Li, Chi Zhang, Sheng Tang, Qinglan Ren \\ Department of Oncology, The First Affiliated Hospital of Chongqing Medical University, Chongqing, China \\ Contributions: (I) Conception and design: Q Ren, Q He; (II) Administrative support: Q Ren; (III) Provision of study materials or patients: Q He, C \\ Zhang; (IV) Collection and assembly of data: Q He, CZ, S Tang; (V) Data analysis and interpretation: Q He, J Li, S Tang; (VI) Manuscript writing: \\ All authors; (VII) Final approval of manuscript: All authors. \\ Correspondence to: Qinglan Ren. No. 1 Youyi Road, Yuanjiagang, Yuzhong District, Chongqing 400016, China. Email: renqlwu@163.com.
}

\begin{abstract}
Background: The treatment of solid malignant tumors using immune checkpoint inhibitors (ICIs) combined with antiangiogenic drugs, has gradually become an active field of clinical research. However, the results are inconsistent. Therefore, we designed this meta-analysis to evaluate the efficacy and safety of ICIs combined with antiangiogenic drugs in patients with solid malignant tumors. We found that the focus of combination therapy studies was on renal cell carcinomas (RCC).
\end{abstract}

Methods: We searched PubMed, Embase, and the Cochrane Library to summarize the hazard ratio (HR) for overall survival (OS), progression-free survival (PFS), and the odds ratio (OR) for objective response rate (ORR), disease control rate (DCR), and adverse events (AEs) of grade 3 or higher.

Results: A total of 6 studies were included. We found that patients who received antiangiogenic drugs combined with ICIs had better OS $[\mathrm{HR}=0.74,95 \%$ confidence interval $(\mathrm{CI}): 0.60-0.89]$, PFS (HR $=0.79$, 95\% CI: $0.70-0.89)$, ORR (OR $=2.34,95 \%$ CI: $1.35-4.04)$ and DCR (OR $=1.52,95 \%$ CI: $1.21-1.91)$ than those without ICIs. There was no significant difference in the incidence of grade 3 or higher AEs $(\mathrm{OR}=0.76$, 95\% CI: 0.52-1.11). Subgroup analyses showed that combination therapy resulted in a lower risk of death in patients with PD-L1 expression $\geq 1 \%$ or $<1 \%$, and better PFS in patients with RCCs who were of different ages, different genders, and different International Metastatic Renal Cell Carcinoma Database Consortium (IMDC) risk scores (favorable/intermediate/poor).

Conclusions: The results of this meta-analysis suggest that ICIs combined with antiangiogenic drugs can provide more clinical benefits to patients with solid malignant tumors without significantly increasing side effects. More clinical trials are needed to validate these findings further.

Keywords: Immune checkpoint inhibitor (ICI); immunotherapy; angiogenesis inhibitor; vascular endothelial growth factor (VEGF); renal cell carcinomas (RCCs)

Submitted May 01, 2020. Accepted for publication Oct 09, 2020.

doi: $10.21037 /$ tcr-20-1975

View this article at: http://dx.doi.org/10.21037/tcr-20-1975

\section{Introduction}

In recent years, with the development of immunotherapy, immune checkpoint inhibitors (ICIs) have gradually changed the treatment pattern of solid malignant tumors. As more ICIs are approved for clinical use, more patients with tumors may obtain a better prognosis. Commonly used ICIs include programmed cell death protein-1 (PD1) inhibitors (such as nivolumab and pembrolizumab), programmed death-ligand 1 (PD-L1) inhibitors (such as atezolizumab, durvalumab, and avelumab), and cytotoxic 
T-lymphocyte-associated antigen 4 (CTLA-4) inhibitors (such as ipilimumab). These ICIs have been widely used for treating various cancers, including lung cancer, renal cell carcinoma (RCC), melanoma, and gastrointestinal tumors (1-5). However, the efficacy of ICI monotherapy for the treatment of solid malignant tumors is limited (6). In order to expand the clinical benefits, the use of combination therapy for cancer treatment is gradually increasing.

Previous research has demonstrated that ICIs, combined with antiangiogenic drugs, can improve the prognosis of certain patients with tumors (7). Antiangiogenic drugs, which are classic anti-tumor agents, are widely used for the treatment of various malignant solid tumors (8). At present, widely used antiangiogenic drugs mainly include tyrosine kinase inhibitors (TKIs), which target the vascular endothelial growth factor (VEGF) signaling pathway (such as sunitinib), and monoclonal antibodies that block VEGF (such as bevacizumab). A previous study demonstrated that antiangiogenic drugs not only promoted the normalization of blood vessels and facilitated the infiltration of lymphocytes into tumor tissues but also down-regulated the number and function of regulatory T-lymphocytes (Tregs), myeloid-derived suppressor cells (MDSCs), and M2 macrophages (9). Also, VEGF reduced the expression of adhesion molecules on endothelial cells, and also reduced the ability of endothelial cells to recognize white blood cells (including tumor-killing $\mathrm{T}$ cells). VEGF can also reduce the expression of PD-1 on killer T cells (10) and inhibit the maturation of dendritic cells (11). Therefore, antiangiogenic drugs, by inhibiting the effects of VEGF, can enhance the anti-tumor ability of the immune system. Researchers have also observed that antiangiogenic drugs combined with PD-1/PD-L1 inhibitors had a synergistic therapeutic effect in a mouse model (12).

Nonetheless, the efficacy and safety of ICIs combined with antiangiogenic drugs in solid malignant tumors are not always consistent (13), and combination therapy remains controversial. We, therefore, performed a metaanalysis to compare the efficacy and safety of antiangiogenic drugs with or without ICIs in solid malignant tumors. We found that combination therapy mainly focused on RCC. We present the following article in accordance with the PRISMA reporting checklist (available at http://dx. doi. org/10. 21037/tcr-20-1975).

\section{Methods}

\section{Search strategy and selection criteria}

We searched PubMed, Embase, and the Cochrane Library for articles before March 2020. We used "immune checkpoint inhibitor; immunotherapy; angiogenesis inhibitor; antiangiogenic drug; VEGF; malignant solid tumor" as keywords. The language was limited to English. The inclusion criteria were designed based on the PICOS principle-population: patients with solid malignant tumors; intervention: antiangiogenic drugs combined with an ICI; comparison: treatment without an ICI; outcomes: overall survival (OS), progression-free survival (PFS), objective response rate (ORR), disease control rate (DCR), adverse events (AEs) of grade 3 or higher; study: randomized controlled trials. Two investigators independently reviewed all articles. The exclusion criteria were as follows: (I) abstract-only papers; (II) not published in English; (III) not a comparative study; (IV) not a randomized trial; (V) not a combination study with an ICI; (VI) did not study solid tumors; (VII) study had no outcomes.

\section{Data extraction and quality assessment}

$\mathrm{QH}$ and $\mathrm{CZ}$ independently extracted the following data sets in each study: the publication year, trial phase, study design, disease, treatments, sample size, median age, and outcomes. If there was a disagreement between the two investigators, a discussion with a third author was conducted to reach a consensus. QH and JL independently used the Cochrane Collaboration's tool to assess the quality of the selected studies using the following ratings: low risk (+), undefined risk (?), and high risk (-).

\section{Statistical analysis}

The meta-analysis was conducted using RevMan 5.3 software (The Nordic Cochrane Center, Cochrane Collaboration). OS and PFS were evaluated through HR. OR evaluated ORR, DCR and AEs of grade 3 or higher. The main results were analyzed in subgroups. All data were extracted from the included studies. When $\mathrm{I}^{2}<33$, a fixed effect (FE) model was used, and when $\mathrm{I}^{2} \geq 33$, a random effect (RE) model was used. The results were illustrated 
using forest plots. Heterogeneity was estimated using Cochran's $\mathrm{Q}$ test and $\mathrm{I}^{2}$ statistics. The significance level was set as $<0.05$. Publication bias was evaluated using a funnel plot, and a sensitivity analysis was performed by removing each study in turn.

\section{Results}

\section{Included studies and study quality}

A total of 1,886 related studies were retrieved from the databases. After eliminating duplicate studies, screening abstracts, and reading full texts, we found eight articles on solid malignant tumors $(7,13-19)$. Table 1 summarizes the main baseline characteristics and survival results of these studies. We found that in these eight articles, only two types of tumors were included. There were six studies on kidney cancer and two on non-small cell lung cancer (NSCLC). Due to the inconsistency of the tumor microenvironment in RCC and NSCLC, after careful consideration, we finally decided to include six studies on RCC in this review (Figure 1). Among these six articles, 1 article (18) was a subgroup analysis of the randomized trials described in another article (13), and we included this subgroup analysis article only in our subgroup analysis. The randomized trials we included were of high quality (Figure 2).

In these six studies, we pooled the HR of OS in 4 trials and PFS in 5 trials. We also pooled the OR of ORR in 5 trials, DCR in 3 trials, and grade 3 or higher AEs in 3 trials. We performed a subgroup analysis of the results of OS, PFS, ORR, and DCR. In addition to the small heterogeneity in the OS group, OS subgroup, and PFS subgroup, there was evident heterogeneity among most studies. Therefore, the FE model was used in the OS group, OS subgroup, and the PFS subgroup, and the RE model was applied for the rest of the groups.

\section{Overall survival}

The pooled HR suggested that ICIs combined with antiangiogenic drugs significantly improved the OS of patients with RCC (HR $=0.74,95 \%$ CI: 0.60-0.89, $\mathrm{I}^{2}=64 \%, \mathrm{n}=4$ ) (Figure 3). As shown in the subgroup analysis (Figure 3), regardless of whether PD-L1 expression was $\geq 1 \%$ or $<1 \%$, ICIs combined with antiangiogenic drugs showed a lower risk of death (HR $=0.76,95 \%$ CI: 0.62-0.92, $\mathrm{n}=4 ; \mathrm{HR}=0.74,95 \%$ CI: $0.60-0.92, \mathrm{n}=2$, respectively).

\section{PFS}

We found that ICIs combined with antiangiogenic drugs significantly prolonged the PFS of patients with RCC (HR $=0.79$; 95\% CI: 0.70-0.89, $\mathrm{I}^{2}=46 \%, \mathrm{n}=5$ ) (Figure 4). Subgroup analyses of PFS indicated that combination therapy with ICIs and antiangiogenic drugs improved PFS in patients with RCC who were of different ages (age $<65$ years: $\mathrm{HR}=0.66,95 \%$ CI: $0.54-0.80$; age $\geq 65$ years: HR $=0.66,95 \%$ CI: 0.51-0.86), different genders (male: $\mathrm{HR}=0.68,95 \%$ CI: $0.57-0.82$; female: $\mathrm{HR}=0.66,95 \%$ CI: 0.49-0.91), different International Metastatic RCC Database Consortium (IMDC) risk scores (favorable/ intermediate/poor) ( $\mathrm{HR}=0.71,95 \% \mathrm{CI}: 0.52-0.96$; HR $=0.71,95 \%$ CI: $0.60-0.84$, HR $=0.58,95 \%$ CI: $0.43-0.79$, respectively), an intermediate/poor Memorial SloanKettering Cancer Center (MSKCC) score (HR $=0.74,95 \%$ CI: $0.64-0.87, \mathrm{HR}=0.55,95 \%$ CI: $0.41-0.74$, respectively), PD-L1 expression $\geq 1 \%$ (HR $=0.67,95 \%$ CI: $0.59-0.76$ ), or underwent nephrectomy (HR $=0.67,95 \%$ CI: 0.55-0.81) (Figure 5).

\section{ORR and DCR}

We summarized the OR of ORR and DCR (Figure 6) and found that both in the PD-L1 $\geq 1 \%$ subgroup and the total population, ICIs combined with antiangiogenic drugs showed better ORR (PD-L $1 \geq 1 \%$ : OR $=2.83,95 \%$ CI: $1.43-5.63, \mathrm{n}=4$; total population: $\mathrm{OR}=2.34,95 \% \mathrm{CI}: 1.35-$ $4.04, \mathrm{n}=5$, respectively) and better DCR (PD-L1 $\geq 1 \%$ : OR $=1.73,95 \%$ CI: $1.12-2.67, \mathrm{n}=3$; total population: $\mathrm{OR}=1.52$, 95\% CI: $1.21-1.91, \mathrm{n}=3$, respectively).

\section{Toxicity}

This meta-analysis evaluated treatment toxicity by analyzing the OR of grade 3 or higher AEs. The outcomes revealed that there was no statistically significant difference in toxicity between the 2 treatments $(\mathrm{OR}=0.76,95 \% \mathrm{CI}$ : $0.52-1.11, \mathrm{P}=0.15, \mathrm{I}^{2}=71 \%, \mathrm{n}=3$ ) (Figure 7).

\section{Publication bias and sensitivity analysis}

Since our analysis included less than ten studies, we did not perform publication bias analysis. Sensitivity analysis was conducted, and the results showed that deleting any study did not significantly change our results, indicating that the 


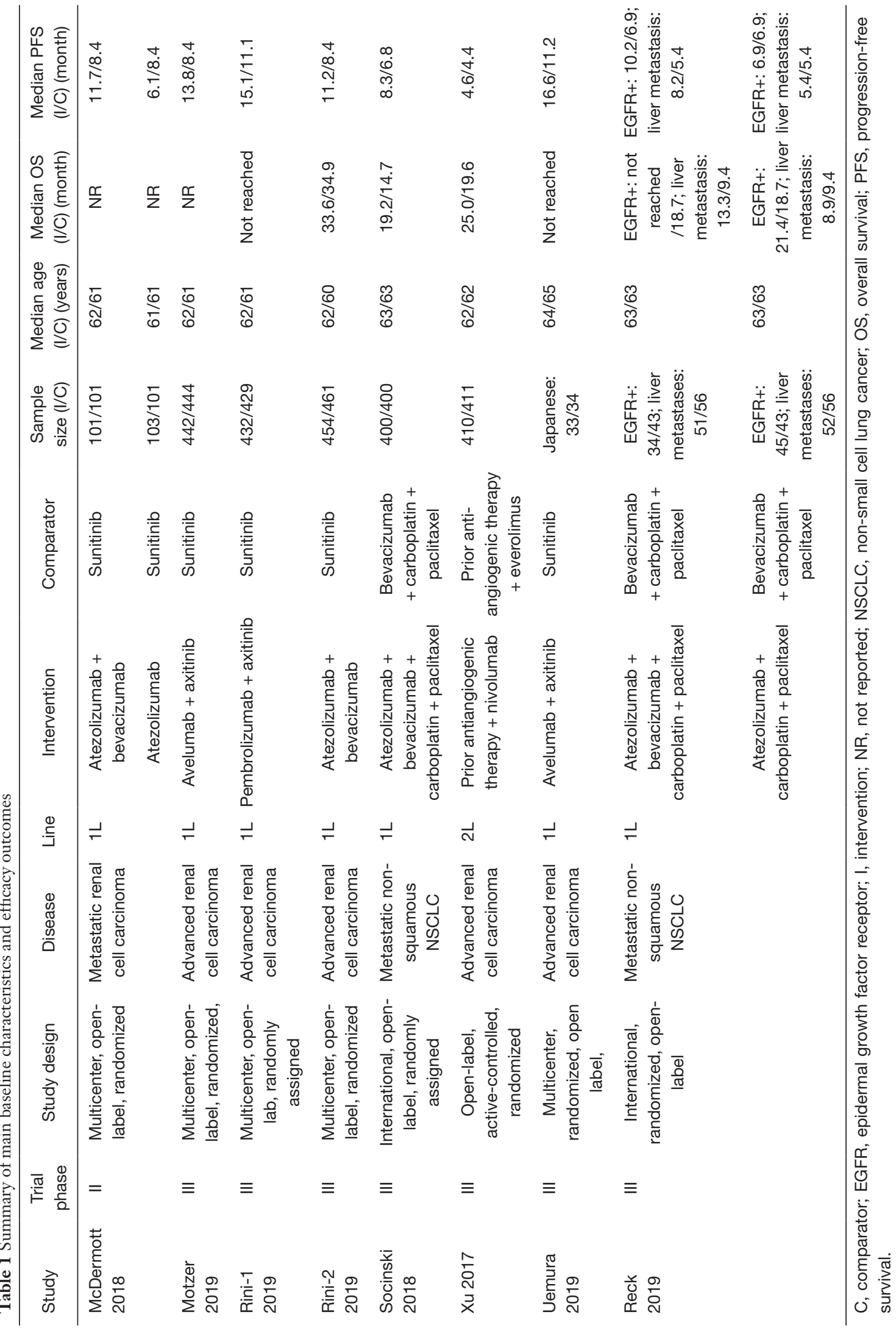




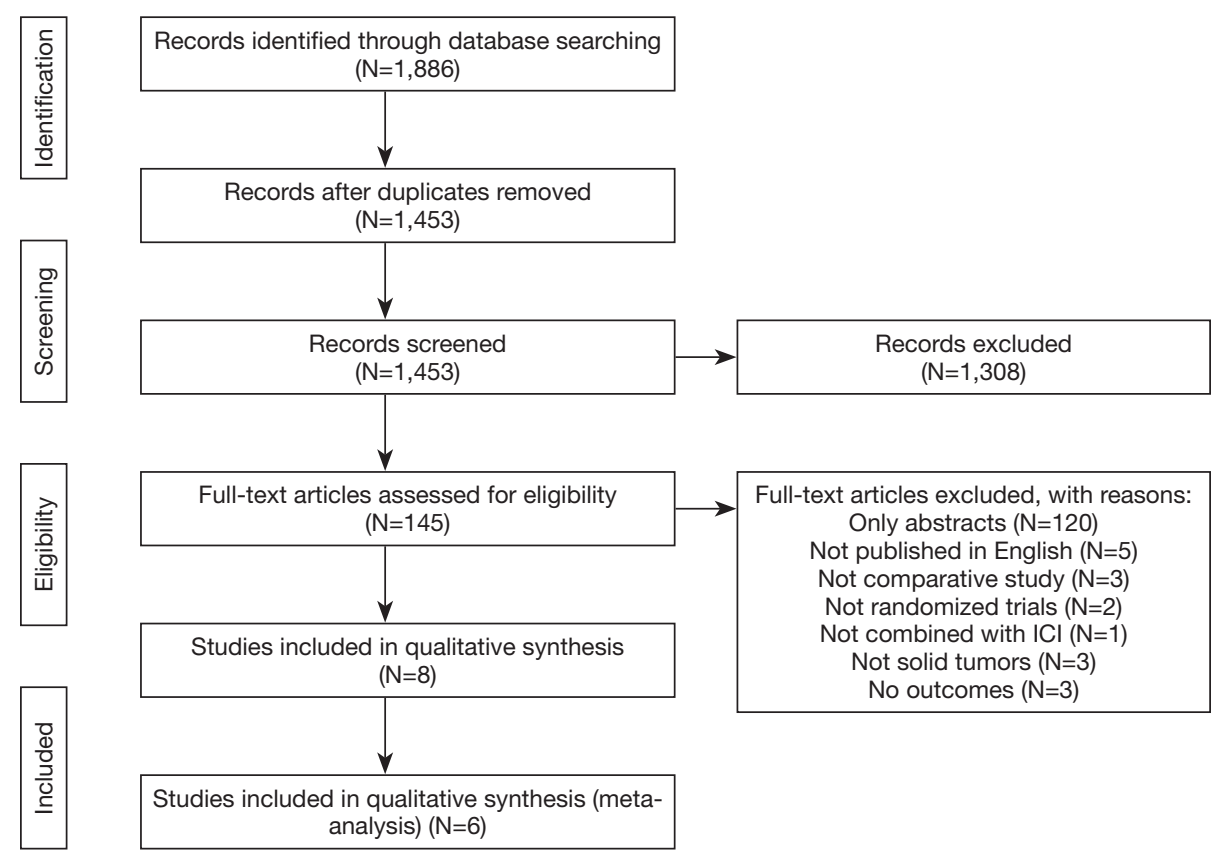

Figure 1 The flow diagram described our process of selection studies. We finally included 6 retrospective cohort studies.

results were robust and credible (Table 2).

\section{Discussion}

The efficacy of immunotherapy monotherapy for solid malignant tumors is far from satisfactory. Some studies have shown that ICIs, combined with antiangiogenic drugs, demonstrate efficacy for solid malignant tumors, and the toxicity is tolerable. However, the outcomes of current studies on combination therapy are inconsistent. A previous meta-analysis examined ICIs in combination with antiangiogenic therapy (20); however, the article did not include a subgroup analysis. Also, a series of studies on ICIs combined with antiangiogenic drugs for the treatment of solid malignant tumors were recently published. Hence, we designed and implemented this meta-analysis. In this meta-analysis, ICIs combined with antiangiogenic drugs improved the OS, PFS, ORR, and DCR of patients with solid malignant tumors, and there was no statistically significant difference in the incidence of grade 3 or higher AEs between the 2 treatments.

Regardless of the expression level of PD-L1, our subgroup analysis showed that patients who received ICIs combined with antiangiogenic drugs had longer OS. Among the patients with solid malignant tumors who had PD-L1 expression $\geq 1 \%$, patients receiving antiangiogenic drugs with ICIs had better PFS, ORR, and DCR than those without ICIs. Notably, 1 study evaluated the effect of nivolumab or everolimus in patients with RCC who previously received antiangiogenic drugs.

In RCC, we found that combination treatment prolonged the PFS of patients with different ages, different genders, different IMDC risk scores (favorable/ intermediate/poor), an intermediate/poor MSKCC score, or patients who underwent nephrectomy. In RCC, Rini et al. also observed that when treated with combination therapy, patients of different ages, different genders, or patients with an intermediate/poor IMDC risk score all had improved OS (7).

In patients with non-squamous NSCLC with epidermal growth factor receptor (EGFR) mutations, Reck et al. found no significant difference in OS and PFS in patients who took antiangiogenic drugs with or without ICIs (19). Interestingly, in NSCLC patients with exon 19 deletion or Leu858Arg mutation, it was observed that combination therapy improved OS and PFS. They also found that for NSCLC patients with EGFR mutations who had previously received TKI treatment, combination therapy improved PFS, but its effect on OS was not statistically different compared to antiangiogenic treatment without an ICI. Also, they found that NSCLC patients with liver metastases treated with combination therapy had a PFS advantage and 

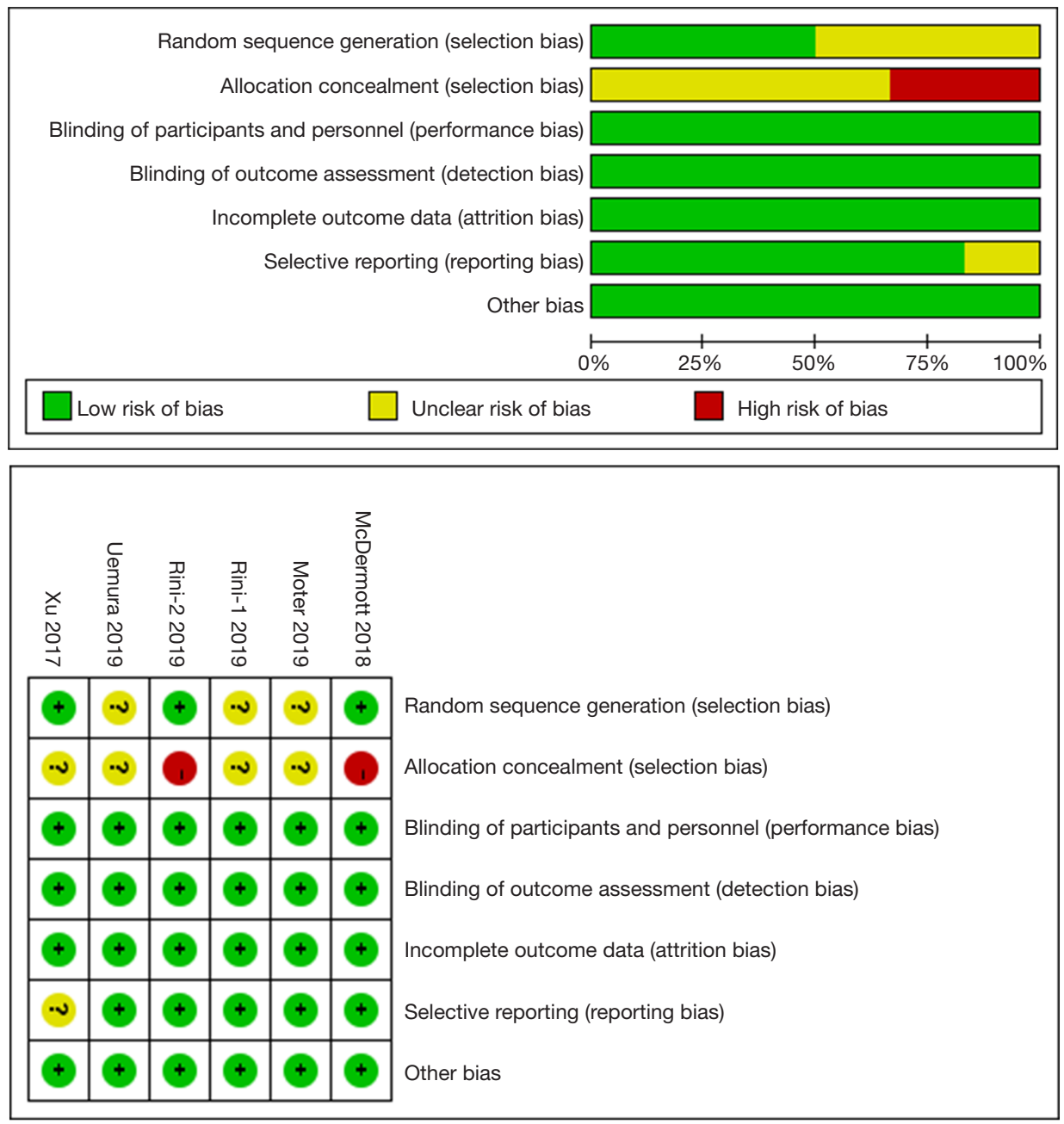

Figure 2 Risk bias of studies included.

a lower risk of death (19).

Some studies have shown that ICIs, combined with antiangiogenic drugs, can increase treatment toxicity (16). However, in this meta-analysis, there was no significant difference in the toxicity of antiangiogenic drugs with or without ICIs. Common adverse effects of combination therapy included neutropenia, hypertension, alopecia, peripheral neuropathy, nausea, anemia, decreased appetite, and diarrhea. Therefore, the implementation of combination therapy necessitates close attention to prevention, early detection, and proper handling of these side effects.

All the articles included in this meta-analysis were randomized controlled trials in RCC patients. We also discussed the role of combination therapy in NSCLC. Our results showed that ICIs, combined with antiangiogenic drugs, were safe and effective. Also, recent studies have found that combination therapy also had a role in other types of tumors. In unresectable hepatocellular carcinoma (uHCC), lenvatinib combined with pembrolizumab demonstrated anti-tumor activity and was well tolerated in patients (21), and atezolizumab combined with bevacizumab also demonstrated better OS and PFS than sorafenib alone (22). Researchers have also found that in advanced hepatocellular carcinoma, gastric cancer, or esophagogastric junction carcinoma, the ORR of SHR-1210 combined with apatinib was $30.8 \%$, and the toxicity was acceptable (23).

Furthermore, previous studies have demonstrated the efficacy of regorafenib combined with nivolumab for advanced colorectal cancer or gastric cancer (24). A 


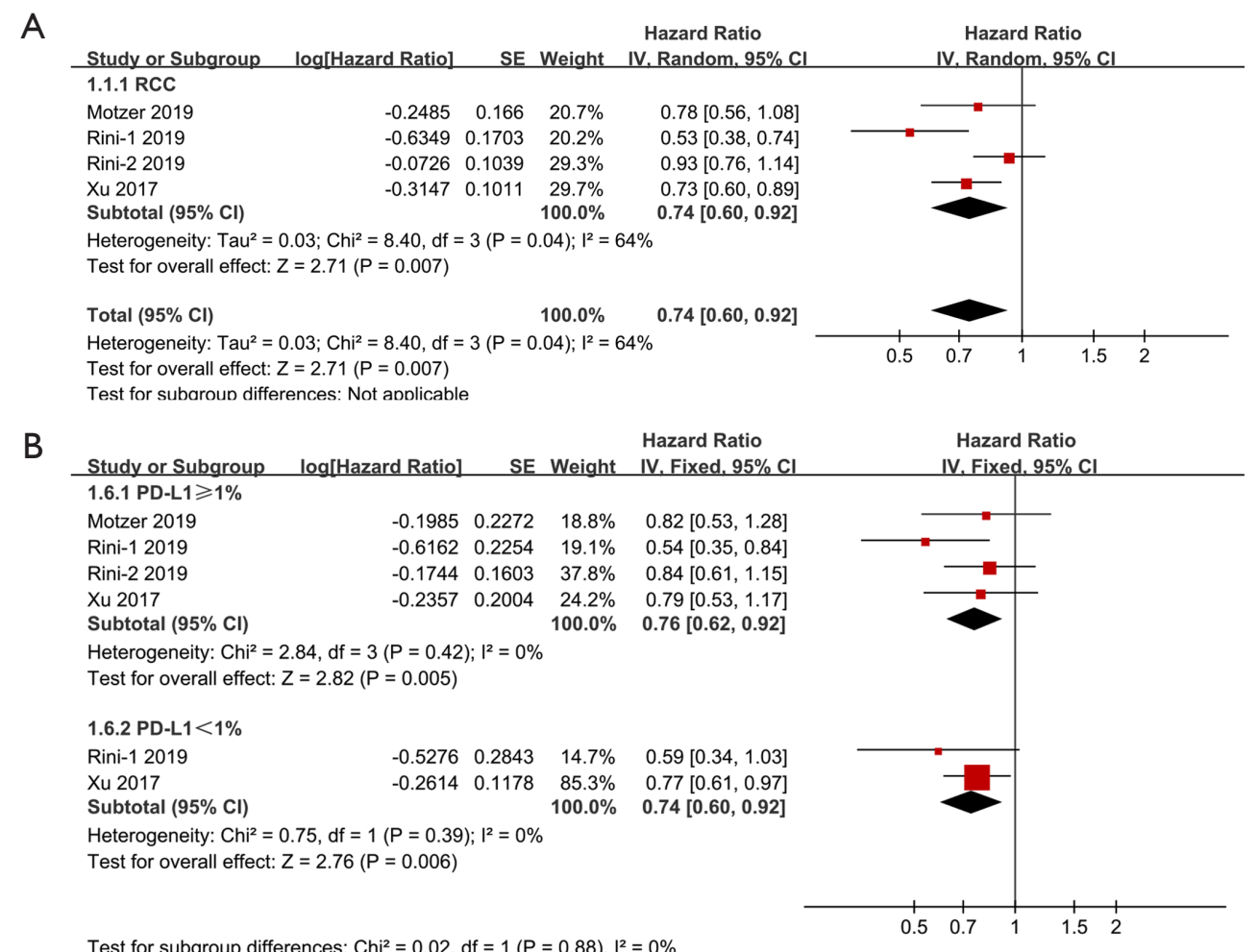

Figure 3 Forest plot for OS and OS subgroup. RCC, renal cell carcinomas.

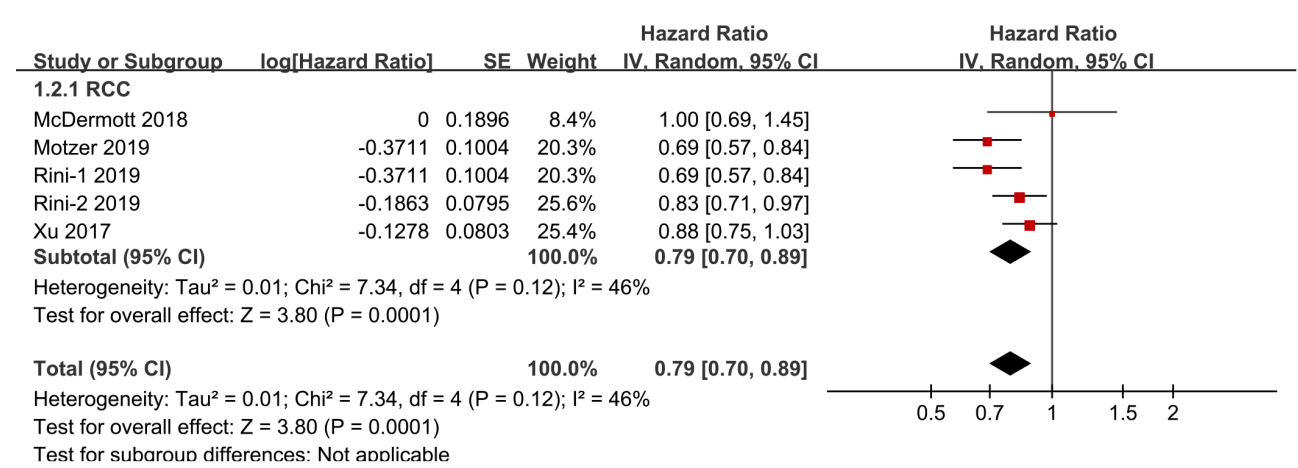

Figure 4 Forest plot for PFS. RCC, renal cell carcinomas.

study also reported that $39.6 \%$ of patients with advanced endometrial carcinoma receiving lenvatinib combined with pembrolizumab had an objective response at 24 weeks (25). In the recurrent epithelial ovarian, fallopian tube, or primary peritoneal cancer, a phase II trial found pembrolizumab combined with bevacizumab and oral metronomic cyclophosphamide had a greater and longerlasting tumor response compared with pembrolizumab monotherapy or bevacizumab combined with oral cyclophosphamide, and the AEs were tolerated (26). Studies have also shown that in patients with advanced sarcomas, axitinib combined with pembrolizumab had preliminary activity and controllable toxicity, especially in the alveolar soft-part sarcoma (27).

Our results indicated that ICIs combined with antiangiogenic drugs were safe and effective for some solid 


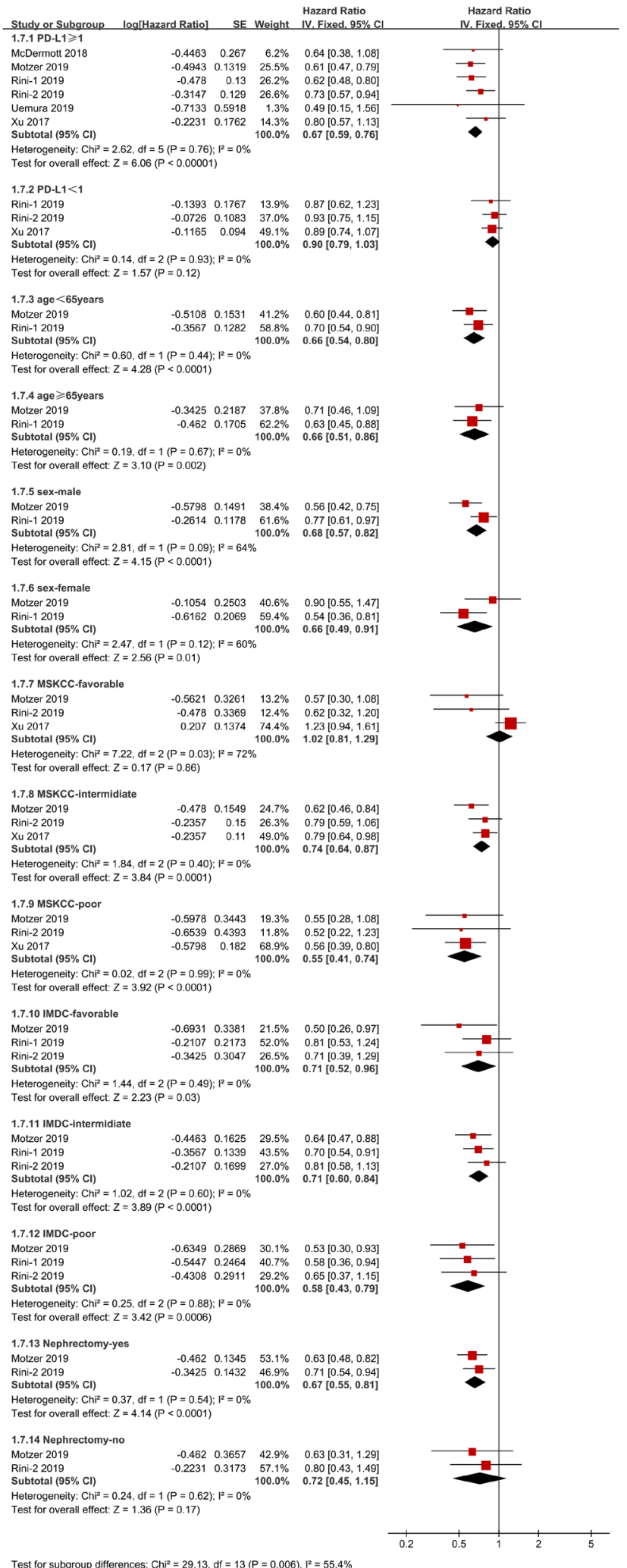

Figure 5 Forest plot for PFS subgroup. MSKCC, Memorial Sloan-Kettering Cancer Center; IMDC, International Metastatic Renal Cell Carcinoma Database Consortium. 


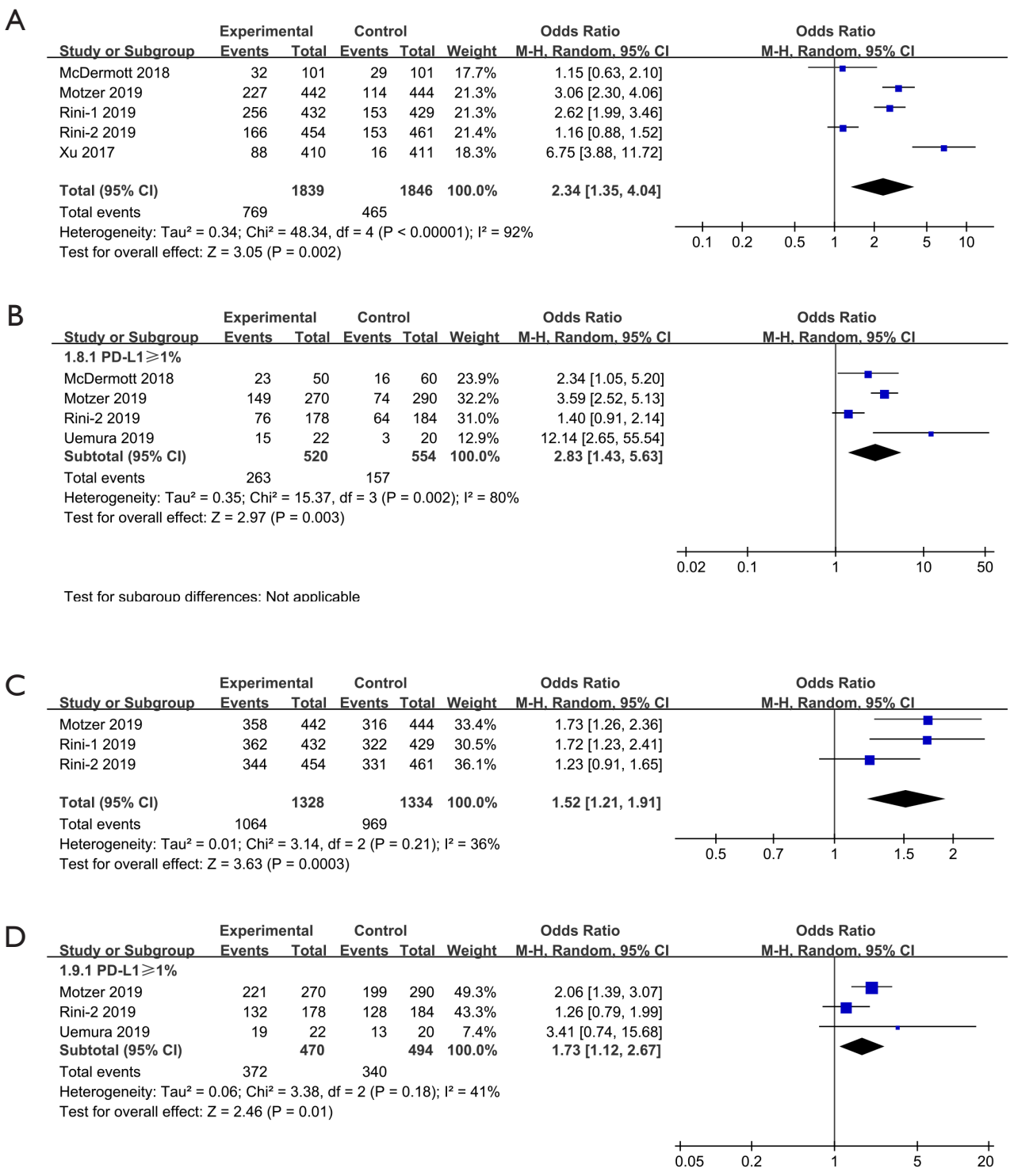

Test for subaroun differences: Not anolicable

Figure 6 Forest plot for ORR (A), ORR by subgroup (B), DCR (C), DCR by subgroup (D). ORR, objective response rate; DCR, disease control rate.

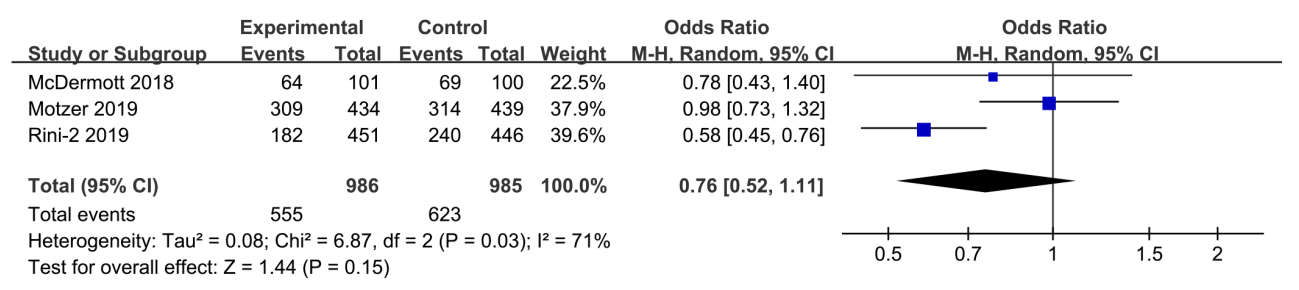

Figure 7 Forest plot for grade $\geq 3$ AEs. AEs, adverse events. 
Table 2 The sensitivity analyses of the study

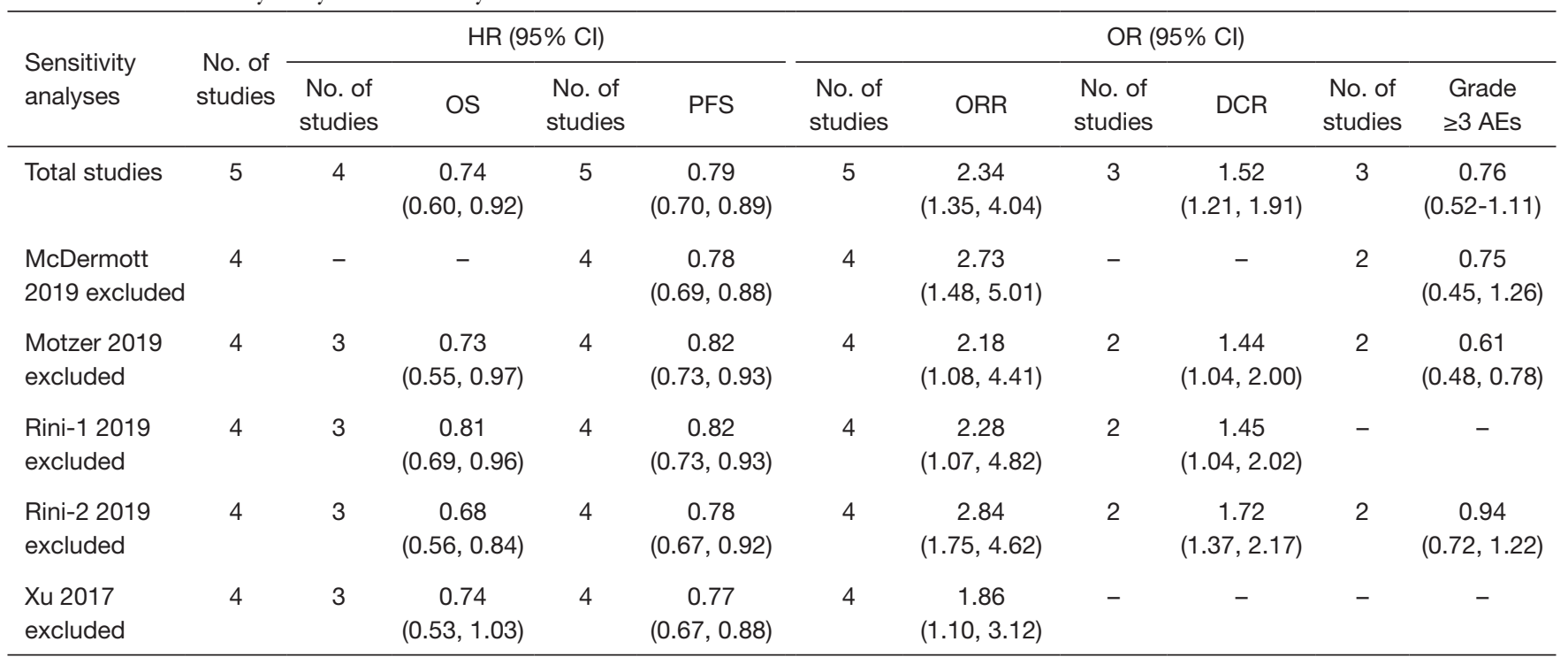

$\mathrm{AE}$, adverse effect; $\mathrm{Cl}$, confidence interval; $\mathrm{DCR}$, disease control rate; HR, hazard ratio; No., number; OR, odds ratio; ORR, objective response rate; OS, overall survival; PFS, progression-free survival.

malignant tumors, suggesting that combination therapy is promising. However, the specific treatment options for different malignant solid tumors need to be further explored in large-scale prospective randomized clinical trials. On the clinicaltrials.gov website, many trials are investigating ICIs combined with antiangiogenic drugs in the treatment of solid malignant tumors. These ICIs include pembrolizumab, nivolumab, ipilimumab, durvalumab, sintilimab, toripalimab, AK105, TSR-042, SHR-1210, HLX10, and BCD-100. Antiangiogenic drugs include bevacizumab, anlotinib, axitinib, apatinib, endostar, and lenvatinib. The tumors studied in these trials range from lung cancer, melanoma, urinary system tumors, head and neck tumors, gastrointestinal tumors, neuroendocrine tumors, hepatobiliary malignancies, ovarian cancer, cervical cancer, and triple-negative breast cancer. With the broadening of future research, ICIs combined with antiangiogenic drugs are expected to bring long-term clinical benefits to more patients with various malignant solid tumors.

\section{Strengths and limitations}

There were some limitations to this study. First, we only included studies on RCC. Furthermore, our results were hypothesis-generating. Hence more trials are needed to evaluate the efficacy and safety of combination therapy in solid malignant tumors. Second, the number of studies and the patients involved in certain subgroups were small. More clinical data from larger-scale studies are required to confirm the benefits of combination therapy. Third, some $\mathrm{I}^{2}$ values in this meta-analysis were large, which meant there was heterogeneity between studies. Additionally, due to the small number of studies included, we did not conduct a publication bias assessment. Some unpublished and missing data may bias the aggregation effect.

\section{Conclusions}

This meta-analysis showed that ICIs combined with antiangiogenic drugs could prolong OS and PFS and improve ORR and DCR in some patients with RCC, with a tolerable toxicity profile. More clinical trials are still needed to further screen for patients who can benefit from the combination therapy and optimize treatment strategies.

\section{Acknowledgments}

Funding: None.

\section{Footnote}

Reporting Checklist: The authors have completed the PRISMA reporting checklist. Available at http:// dx. doi. org/10. 21037/tcr-20-1975 
Conflicts of Interest: All authors have completed the ICMJE uniform disclosure form (available at http://dx. doi. org/10.21037/tcr-20-1975). The authors have no conflicts of interest to declare.

Ethical Statement: The authors are accountable for all aspects of the work in ensuring that questions related to the accuracy or integrity of any part of the work are appropriately investigated and resolved.

Open Access Statement: This is an Open Access article distributed in accordance with the Creative Commons Attribution-NonCommercial-NoDerivs 4.0 International License (CC BY-NC-ND 4.0), which permits the noncommercial replication and distribution of the article with the strict proviso that no changes or edits are made and the original work is properly cited (including links to both the formal publication through the relevant DOI and the license). See: https://creativecommons.org/licenses/by-nc-nd/4.0/.

\section{References}

1. Li S, Zhang S, Liu J, et al. The effect of PD-L1/PD-1 immunotherapy in the treatment of squamous non-smallcell lung cancer: a meta-analysis of randomized controlled clinical trials. J Thorac Dis 2019;11:4453-63.

2. Nishijima TF, Shachar SS, Nyrop KA, et al. Safety and Tolerability of PD-1/PD-L1 Inhibitors Compared with Chemotherapy in Patients with Advanced Cancer: A MetaAnalysis. Oncologist 2017;22:470-9.

3. Yun S, Vincelette ND, Green MR, et al. Targeting immune checkpoints in unresectable metastatic cutaneous melanoma: a systematic review and meta-analysis of anti-CTLA-4 and anti-PD-1 agents trials. Cancer Med 2016;5:1481-91.

4. Marra A, Viale G, Curigliano G. Recent advances in triple negative breast cancer: the immunotherapy era. BMC Med 2019;17:90.

5. Puzzoni M, Silvestris N, Leone F, et al. The Immune Revolution in Gastrointestinal Tumours: Leading the Way or Just Following? Target Oncol 2016;11:593-603.

6. Yang Y, Jin G, Pang Y, et al. Comparative Efficacy and Safety of Nivolumab and Nivolumab Plus Ipilimumab in Advanced Cancer: A Systematic Review and MetaAnalysis. Front Pharmacol 2020;11:40.

7. Rini BI, Plimack ER, Stus V, et al. Pembrolizumab plus Axitinib versus Sunitinib for Advanced Renal-Cell Carcinoma. N Engl J Med 2019;380:1116-27.
8. Roviello G, Bachelot T, Hudis CA, et al. The role of bevacizumab in solid tumours: A literature based meta-analysis of randomised trials. Eur J Cancer 2017;75:245-58.

9. Gao F, Yang C. Anti-VEGF/VEGFR2 Monoclonal Antibodies and their Combinations with PD-1/PDL1 Inhibitors in Clinic. Curr Cancer Drug Targets 2020;20:3-18.

10. Voron T, Colussi O, Marcheteau E, et al. VEGF-A modulates expression of inhibitory checkpoints on CD8+ T cells in tumors. J Exp Med 2015;212:139-48.

11. Chen DS, Hurwitz H. Combinations of Bevacizumab With Cancer Immunotherapy. Cancer J 2018;24:193-204.

12. Meder L, Schuldt P, Thelen M, et al. Combined VEGF and PD-L1 Blockade Displays Synergistic Treatment Effects in an Autochthonous Mouse Model of Small Cell Lung Cancer. Cancer Res 2018;78:4270-81.

13. Motzer RJ, Penkov K, Haanen J, et al. Avelumab plus Axitinib versus Sunitinib for Advanced Renal-Cell Carcinoma. N Engl J Med 2019;380:1103-15.

14. McDermott DF, Huseni MA, Atkins MB, et al. Clinical activity and molecular correlates of response to atezolizumab alone or in combination with bevacizumab versus sunitinib in renal cell carcinoma. Nat Med 2018;24:749-57.

15. Rini BI, Powles T, Atkins MB, et al. Atezolizumab plus bevacizumab versus sunitinib in patients with previously untreated metastatic renal cell carcinoma (IMmotion151): a multicentre, open-label, phase 3, randomised controlled trial. Lancet 2019;393:2404-15.

16. Socinski MA, Jotte RM, Cappuzzo F, et al. Atezolizumab for First-Line Treatment of Metastatic Nonsquamous NSCLC. N Engl J Med 2018;378:2288-301.

17. Xu JX, Maher VE, Zhang L, et al. FDA Approval Summary: Nivolumab in Advanced Renal Cell Carcinoma After Anti-Angiogenic Therapy and Exploratory Predictive Biomarker Analysis. Oncologist 2017;22:311-7.

18. Uemura M, Tomita $Y$, Miyake H, et al. Avelumab plus axitinib vs sunitinib for advanced renal cell carcinoma: Japanese subgroup analysis from JAVELIN Renal 101. Cancer Sci 2020;111:907-23.

19. Reck M, Mok TSK, Nishio M, et al. Atezolizumab plus bevacizumab and chemotherapy in non-small-cell lung cancer (IMpower150): key subgroup analyses of patients with EGFR mutations or baseline liver metastases in a randomised, open-label phase 3 trial. Lancet Respir Med 2019;7:387-401.

20. Wei Y, Du Q, Jiang X, et al. Efficacy and safety of 
combination immunotherapy for malignant solid tumors: A systematic review and meta-analysis. Crit Rev Oncol Hematol 2019;138:178-89.

21. A phase $1 \mathrm{~b}$ trial of lenvatinib (LEN) plus pembrolizumab $(\mathrm{PEM})$ in patients (pts) with unresectable hepatocellular carcinoma (uHCC). Available online: https://ascopubs.org/ doi/abs/10.1200/jco.2018.36.15_suppl.4076

22. Cheng AL, Qin S, Ikeda M, et al. IMbrave150: Efficacy and safety results from a ph III study evaluating atezolizumab (atezo) 1 bevacizumab (bev) vs sorafenib (Sor) as first treatment (tx) for patients (pts) with unresectable hepatocellular carcinoma (HCC). Ann Oncol 2019;30:ix186-ix187.

23. Xu J, Zhang Y, Jia R, et al. Anti-PD-1 Antibody SHR-1210 Combined with Apatinib for Advanced Hepatocellular Carcinoma, Gastric, or Esophagogastric Junction Cancer: An Open-label, Dose Escalation and Expansion Study. Clin Cancer Res 2019;25:515-23.

24. Fukuoka S, Hara H, Takahashi N, et al. Regorafenib

Cite this article as: $\mathrm{He} \mathrm{Q}$, Li J, Zhang C, Tang S, Ren Q. The efficacy and safety of immune checkpoint inhibitors combined with antiangiogenic drugs in renal cell carcinomas: a systematic review and meta-analysis. Transl Cancer Res 2020;9(11):67806791. doi: 10.21037/tcr-20-1975
Plus Nivolumab in Patients With Advanced Gastric or Colorectal Cancer: An Open-Label, Dose-Escalation, and Dose-Expansion Phase Ib Trial (REGONIVO, EPOC1603). J Clin Oncol 2020;38:2053-61.

25. Makker V, Rasco D, Vogelzang NJ, et al. Lenvatinib plus pembrolizumab in patients with advanced endometrial cancer: an interim analysis of a multicentre, open-label, single-arm, phase 2 trial. Lancet Oncol 2019;20:711-8.

26. Zsiros E, Frederick PJ, Akers SN, et al. A phase II trial of pembrolizumab in combination with bevacizumab and oral metronomic cyclophosphamide for recurrent epithelial ovarian, fallopian tube or primary peritoneal cancer. Available online: https://www.gynecologiconcology-online. net/article/S0090-8258(19)30554-2/fulltext

27. Wilky BA, Trucco MM, Subhawong TK, et al. Axitinib plus pembrolizumab in patients with advanced sarcomas including alveolar soft-part sarcoma: a single-centre, single-arm, phase 2 trial. Lancet Oncol 2019;20:837-48. 